\title{
Interrogantes en la prospección de Papanicolaou
}

\author{
Dr. Ramiro A. Chadid H.*
}

\section{INTRODUCCION}

Este trabajo tiene como fines valorar la incidencia de las displasias y del cáncer de cérvix en la población que concurre al Hospital General de Barranquilla y el grado de certeza diagnóstico de la citología de Papanicolaou, en el Departamento de Anatomía Patológica y Citología del mismo hospital.

\section{ANTECEDENTES}

A pesar de que la primera comunicación de Papanicolaou data de 1928, no fue sino hasta después de la segunda guerra mundial cuando el método de diagnóstico citológico del cáncer del cervix ganó la aprobación médica. Desde entonces ha sido signo de buen funcionamiento hospitalario el poseer centros de pesquisa del cáncer cervical a través de dicho método.

La difusión del citodiagnóstico permitió no solo confirmar la incidencia del Ca cervical, sino también pronosticarla. El conocimiento de las enfermedades displásicas precancerosas y su evolución, conocimiento debido en parte al mismo

\footnotetext{
* Profesor de Ginecología Universidad Metro-
} politana y Hospital General de Barranquilla. método, ha permitido hacer desaparecer las cifras tradicionales de incidencia del Ca infiltrante e in situ en las localidades, como lo demostraron God $y$ Koch en Frederiksberg, municipio de Copenhague(1).

Las cifras estadísticas que demuestran que casi la mitad de los cánceres de la mujer en los países subdesarrollados, son Ca de cervix (2), ha obligado a los gobiernos a mantener servicios de citodiagnóstico permanentes en los hospitales del estado, con un criterio igual que para las enfermedades infecto-contagiosas.

La incidencia del cáncer de cervix, que llega según el Servicio de Salud Pública de E. U. A. a 97.6 por cada 100 mil habitantes para la población de Puerto Rico (3), y de 1 por 100 habitantes para la población de consulta externa del Hospital Johns Hopkins (4) cuya población de consulta es mayoría de raza negra. Igualmente la incidencia de las displasias cervicales que pueden llegar según Williams (5) al $0.5 \%$ y según Terzano (6) al 6.3\%, nos da el primer interrogante ya que las estadísticas de nuestro país, si no ausentes, son fragmentarias $y$ poco confiables.

La excesiva confianza diagnostica dada al método en nuestro medio, que 
en los buenos Centros oscila entre $60 \%$ (Balaguero) (7) y 95\% (Von Ham) (8) que exige un personal médico $y$ paramédico altamente calificado, lo mismo que la ausencia de autocrítica de la calidad del trabajo realizado nos ofrece otro interrogante que se suma al problema.

De otro lado el hecho demostrado por Kashgarian (9) en el condado de Sheby de Memphis (Tennessee) en donde ofreciendo citologías gratuitas y apoyados por una propaganda bien dirigida en cinco años, sólo lograron despertar el interés del $20 \%$ de la población femenina en repetir anualmente dicho examen, comprueba lo complejo de la utilidad pública de la citología y agrega otro interrogante en la lucha contra el cancer cervical.

Con estos interrogantes, parecía agotarse nuestra capacidad de asombro. Este trabajo es para presentar otro interrogante que no buscabamos como objetivo, pero que nos revela una vez más nuestra idiosincrasia.

\section{MATERIAL Y METODOS}

Se revisaron cuidadosamente los archivos del Departamento de Anatomía Patológica y Citológica del Hospital General de Barranquilla entre enero de 1978 y diciembre de 1982 que comprende cinco años de trabajo de ese Departamento.

Se analizaron todas las citologías ginecológicas y se aislaron aquellas diag nosticadas como sospechosas y positivas (clasificación de papanicolaou III, IV, V) y que ameritaban biopsia subsecuente.

Se revisaron luego los archivos de Anatomía Patológica buscando por el número de registro, el nombre de la paciente o por el renglón de biopsias ginecológicas, la relación entre el estudio citológico y el biopsico. Luego se compararon ambos diagnósticos. Los datos son:

\section{Año 1978}

Total de consultas del Hospital

Total de citologías practicadas

\subsection{6}

Diagnosticadas sospechosas: 71

"

positivas:

20

Confirmadas por Biopsia:

$\begin{array}{lll}3 & \text { sospechosas: } & 2 \mathrm{ca} \\ 3 & \text { positivas: } & 3 \mathrm{ca}\end{array}$

\section{Año 1979}

Total de Consultas del Hospital

87.771
Total de citologías practicadas

$$
5.920
$$

Diagnósticadas sospechosas: 65

" positivas:

22

Confirmadas por Biopsia

11 sospechosas: $3 \mathrm{ca}$

5 positivas: $5 \mathrm{ca}$ 


\section{Año 1980}

Total de Consultas del Hospital:

Total de citologías prácticadas

$$
4.680
$$

Diagnósticadas sospechosas: 25

positivas:

9

Confirmadas por Biopsia:

$\begin{array}{lll}4 & \text { sospechosas: } & 2 \mathrm{ca} \\ 5 & \text { positivas: } & 5 \mathrm{ca}\end{array}$

\section{Año 1981}

Total de Consultas del Hospital

74.599

Total de citologías practicadas

$$
2.973
$$

Diagnósticadas sospechosas: 22

Diagnósticadas positivas:

Confirmadas por Biopsia:

$$
\begin{array}{lll}
3 & \text { sospechosas: } & 1 \mathrm{ca} \\
3 & \text { positivas: } & 2 \mathrm{ca}
\end{array}
$$

\section{Año 1982}

Total de consultas del Hospital

83.263

Total de citologías practicadas

Diagnósticadas sospechosas: 57

$$
\text { positivas: }
$$

Confirmadas por Biopsia

12 sospechosas: $6 \mathrm{ca}$

11 positivas: $8 \mathrm{ca}$
Total de Totales: $1978-1982$

Consultas del Hospital:

424.275

Citologías practicadas

22.816

Diagnósticadas sospechosas: 240

" positivas

85

Confirmadas por Biopsia:

33 sospechosas: $14 \mathrm{ca}$

27 positivas: $23 \mathrm{ca}$

Dejadas de confirmar:

207 sospechosas

58 positivas

Total de Citoloqías

$\begin{array}{lrr}\text { para Biopsiar: } & 325 & 100 \% \\ \text { Biopsadas: } & 60 & 18 \%\end{array}$

Certitud Diagnósticada

Para sospechosas: $40 \%$

" positivas:

$95 \%$

\section{Probabilidad Diagnostica en Ias Pacientes Perdidas}

Sospechosas: $207 \quad(40 \%) \quad 82 \mathrm{ca}$ Positivas: $\quad 55 \quad$ (95\%) $50 \mathrm{ca}$

Una aproximación a la incidencia del cancer de cervix en la consulta externa total (hombre - mujeres y niños) del Hospital General de Barranquilla.

Total de consultas 5 años

424.275

Total de Ca Probables 169 Incidencia: 39.8 por cada 100.000 consultas 
Una aproximación a la incidencia del ca de cervix por citologías practicadas.
Total de citologías

22.816

Total de Ca probables

169
Incidencia: 7.4 por 1000 citologías

Análisis de dos tiempos en la muestra:

Total de pacientes de consultas

$1978-19792$ años.

\section{\begin{tabular}{lll}
177.883 & Citología & 12.166 \\
\hline
\end{tabular}}

Total de pacientes de consulta

1981 - 19822 años

157.862 citología 5.970

\section{CONCLUSIONES}

1. La incidencia del $\mathrm{Ca}$ cervical en la consulta general del Hospital de Barranquilla, es de aproximadamente 40 por cada 100.000 consultas y de 0.8 por cada 100 citologías practicadas.

2. La certeza diagnóstica oscila en el $65 \%$, o sea que el error diagnostico es de $35 \%$, referidos unicamente como falsos positivos, ya que el trabajo no analiza los falsos negativos.

3. El uso de la clasificación de Papanicolaou no permite valorar la certeza del diagnóstico citológico de las displasias cervicales.

4. De las 325 citologías diagnosticadas como probables de cancer, resultado de 5 años de trabajo, sólo 60 de ellas fueron corroboradas por biopsia.
5. Que nadie sabe que se hicieron las otras 205 pacientes que en base a la certeza diagnóstica analizada son 132 canceres de cervix, que fueron diagnosticados por el Hospital, pero cuya evolución y destino no nos fue dable conocer.

6. Que sintetizando, el Hospital General de Barranquilla perdió el $82 \%$ de la labor realizada durante 5 años en la prevención del Ca cervical por el método de Papanicolaou.

7. Que el descenso a la mitad del número de citologías practicadas por año $(1978$ - 79 y $1981-82)$ analizadas en 2 tiempos de la muestra, sin que haya habido un descenso similar en la consulta, indica lo perjudicial que son las decisiones tomadas sin un correcto criterio médico.

\section{RESUMEN}

El trabajo trazado para valorar la incidencia de las displasias y del cáncer de cérvix en la población de consulta del Hospital General de Barranquilla, lo mismo que para valorar la certeza diagnóstica del método en el Departamento de Anatomía Patológica y Citología de dicho Hospital hace revisar al autor los archivos del Hospital referentes al tema. Los hallazgos constatan que por razones ajenas al método se perdió el $82 \%$ del trabajo realizado por dicho Departamento durante los años 1978 1982.

Basado en este hecho se analizan los aspectos relacionados con este método de seguimiento del cáncer de cérvix y se agrega un nuevo interrogante a los otros ya existentes; interrogantes que limitan la trascendencia e importancia de la utilidad pública de la citología. 


\section{INTERROGATIONS REGARDING PAPANICOLAOU PROSPECTION}

\section{SUMMARY}

With the purpose of assesing the method used to detect the incidence of displasia and cervical cancer among the population that is served by Barranquilla General Hospital, and to establish diagnostic accuracy at the Department of Pathology and Cytology of that Hospital, the author studied hospital files on that subject. Findings revealed that

\section{BIBLIOGRAFIA}

1. Citado de NOVAK. Tratado de Ginecología 9a. edición Pág. 263.

2. Citado de KASER. Ginecología y Obstetricia $1 a_{\circ}$ edición 30.353.

3. HAENSZEL. HILHOUSE J. Nat. Cancer Inst. 22: 1157. 1959.

4. NOVAK. Villa Santa Jama. 174: 1395, 1960.
$82 \%$. of the work conducted by the Department between 1978 and 1982 was lost for reasons not related to the method.

Based on these facts, the author analyzes diverse aspects related to the mentioned method of follow-up of cervical cancer and poses new questions on the subject; questions that furtherly limit the importance and validity of cytologies at public hospitals.

5. WILLIAMS HESTER. A. Journal Obst. Gynec. 88: 1029. 1964.

6. TERZANO, Act. Cytol 1: 28, 29 etc. 1957.

7y Citado de JIMENEZ AYALA: Citopato8 logía Ginecológica Tomo I. Pág. 124.

9. KASHGARIAN. ERIKSON Act. Cytol. 10: 111966.

10. Archivo Estadístico: Seccional de Salud del Atlántico. 